\title{
PERFIL EPIDEMIOLÓGICO DOS CASOS DE ESCORPIONISMO NA REGIÃO NORDESTE
}

\section{EPIDEMIOLOGICAL PROFILE OF SCORPIONISM CASES IN THE NORTHEAST REGION}

\author{
Raquel Costa e Silva ${ }^{1}$ \\ Allana Renally Cavalcante Santos de Moraes $^{2}$ \\ Edenilson Cavalcante Santos ${ }^{3}$
}

RESUMO: As modificações no ambiente produzidas pelo homem ocasionam maior contato dos animais peçonhentos com os humanos. OBJETIVO: Apresentar o perfil epidemiológico dos acidentes escorpiônicos na Região Nordeste em 2017. MÉTODO: Realizou-se uma pesquisa transversal, de caráter quantitativo, qualitativo e documental. Os resultados foram obtidos a partir dos dados notificados na plataforma Datasus sobre a ocorrência de acidentes escorpiônicos no Brasil em 2017, especificamente na Região Nordeste. RESULTADOS: Foram notificados 56.100 casos de escorpionismo na Região Nordeste. As mulheres foram as maiores vítimas (56,75\%; $n=31.841)$, a faixa etária entre 20 a 39 anos $(17.696 ; 31,5 \%)$ foi a mais afetada. A maioria dos casos foi identificada como "leve" ( $n=50.094 ; 89,3 \%$ ). Em relação ao número de casos, o Estado de Pernambuco foi o responsável pela maioria das notificações ( $n=14.668 ; 26,1 \%)$. O maior número de óbitos foi no estado da Bahia (16; 11,4\%); Alagoas apresentou a maior incidência, com cerca de 267 casos a cada 100.000 habitantes, e o Maranhão apresentou a maior taxa de letalidade, com 36,1 \%. CONCLUSÃO: Os acidentes por animais peçonhentos aumentam o número e o tempo de internação nos serviços de saúde, acarretando em alta ocupação de leitos, sobrecarga dos profissionais e resultando em maiores gastos para o Sistema Único de Saúde (SUS). É possível observar a importância de estudos subsequentes, para que assim seja possível definir medidas de controle dos acidentes escorpiônicos.

Palavras chave: Epidemiologia; Tityus; Toxicologia.

\footnotetext{
${ }^{1}$ Acadêmica do Curso de Farmácia Generalista - Universidade Estadual da Paraíba.

2 Acadêmica do Curso de Enfermagem - Universidade Estadual da Paraíba.

${ }^{3}$ Médico. Hospital de Emergência e Trauma Dom Luis Gonzaga Fernandes - HETDLGF-CG.
} 
ABSTRACT: The changes in the environment produced by man cause greater contact of venomous animals with humans. Objective: To present the epidemiological profile of scorpion accidents in the Northeast Region in 2017. Method: A survey was performed, is a cross-sectional, quantitative, qualitative and documentary. The results were obtained from data reported in the Datasus platform about the occurrence of scorpion accidents in Brazil in 2017, specifically in the Northeast Region. Results: 56,100 cases of scorpionism were notified in the Northeast. Women were the biggest victims $(56.75 \% ; n=31,841)$, the age group between 20 and 39 years $(17,696 ; 31.5 \%)$ was the most affected. The most cases were identified as "mild" ( $n=50,094 ; 89.3 \%)$. Regarding the number of cases, the state of Pernambuco was responsible for most notifications ( $n=14,668 ; 26.1 \%)$. The highest number of deaths was in the state of Bahia (16; 11.4\%); Alagoas had the highest incidence, with about 267 cases per 100,000 habitants, and Maranhão had the highest case lethality rate, with $36.1 \%$. Conclusion: The accidents caused by venomous animals increase the number and hospitalization time in health services, resulting in high occupancy of hospital beds, overloading professionals and resulting in higher expenses for the Health Unic System (SUS). It is possible to observe the very importance of subsequent studies, so that it is possible to define measures to control scorpion accidents.

Keywords: Epidemiology; Tityus; Toxicology. 


\section{INTRODUÇÃO}

O Brasil possui uma alta diversidade de biomas, sendo um dos países com grande biodiversidade de animais peçonhentos e venenosos. São alguns animais peçonhentos brasileiros de interesse médico - àqueles com relevância para a saúde pública devido ao potencial de gravidade dos acidentes causados, algumas espécies de serpentes, escorpiões, aranhas, lagartas e abelhas. Esse tipo de agravo é caracterizado como emergência clínica, principalmente quando ocorrem em extremos de idade, como em crianças e idosos (BRASIL, 2001; SANTOS et al., 2010; BARRETO et al., 2010; MESCHIAL et al., 2013).

Dentre esses animais, são apontados como os maiores causadores de acidentes no Brasil, alguns tipos de artrópodes (escorpiões e aranhas), serpentes, certos himenópteros (abelhas, vespas, formigas e marimbondos), quilódopos (lacraias) e larvas de lepidópteros urticantes (lagartas-de-fogo) (OLIVEIRA; COSTA; SASSI, 2013). Os acidentes escorpiônicos constituem um problema de saúde pública no Brasil, devido à sua elevada incidência em todas as regiões do país, principalmente porque estes acidentes podem proporcionar quadros clínicos fatais, principalmente em crianças e idosos (ALBUQUERQUE et al., 2015; SANTOS et al., 2010).

Os animais peçonhentos são aqueles capazes de produzir toxina, ou uma mistura delas, por uma glândula, e quando inoculada em outro ser, através de um aparato inoculador natural (espinhos, dentes, aguilhões ou ferrões), podem alterar o funcionamento do metabolismo da vítima (BRASIL, 2019).

Acidentes envolvendo estes animais acarretam impactos físicos, sociais e econômicos significativos. Porém, mesmo com altas taxas de incidência ao longo dos anos, este cenário se configura como um dos problemas de saúde mais negligenciados no mundo, por não fazer parte das ações prioritárias dos governos (CARMO et al., 2016). 


\section{METODOLOGIA}

O presente estudo trata-se de uma pesquisa transversal, de caráter quantitativo, qualitativo e documental, sendo realizada no último semestre de 2019. Os resultados foram obtidos a partir dos dados notificados na plataforma Datasus (Departamento de Informática do Sistema Único de Saúde), sobre a ocorrência de acidentes com animais peçonhentos no Brasil no período 2017, e especificamente nos nove estados que compõem o Nordeste, já que esta é reconhecidamente a região que concentra o maior número de casos no país. Como este agravo trata-se de uma notificação compulsória em todo o território nacional, os dados foram obtidos a partir das notificações feitas para o Ministério da Saúde.

As variáveis analisadas foram o número de casos para cada estado e para o país, bem como o número de óbitos ocorridos. Os resultados foram submetidos à

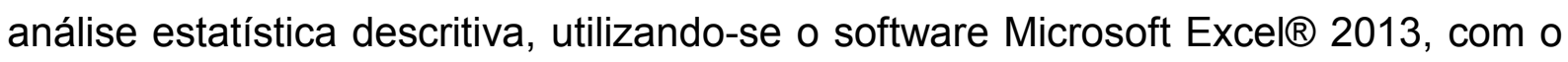
suplemento da ferramenta de análises de dados VBA (Visual Basic for Application), onde foram calculadas as taxas de incidência e letalidade, obtendo distribuições absolutas das variáveis nominais e a média das variáveis numéricas.

A taxa de incidência destes casos foi determinada como a razão entre o número de casos notificados e a estimativa da população, multiplicado por 100.000, o equivalerá a casos entre 100.000 habitantes:

$$
I=\frac{\text { Número de Casos Notificados }}{\text { Estimativa de número de habitantes }}=x 100.000
$$

De semelhante modo, a taxa de letalidade foi obtida através da razão entre número de óbitos pelo número de casos notificados:

$$
L=\frac{\text { Número de Óbitos }}{\text { Número de Casos Notifcads }}=x 100
$$




\section{REFERENCIAL TEÓRICO}

Os escorpiões surgiram há mais de 400 milhões de anos, sobrevivendo devido à sua capacidade evolutiva e adaptativa (BRASIL, 2009). A fauna escorpiônica brasileira é representada por quatro famílias - Bothriuridae, Chactidae, Liochelidae e Buthidae, sendo esta última responsável por representar $60 \%$ do total, incluindo as espécies de interesse em saúde pública (BRASIL, 2009).

A espécie Tityus stigmurus é responsável pela maioria dos acidentes no Nordeste. Assemelha-se ao T. serrulatus nos seus hábitos e coloração, porém apresenta uma faixa escura longitudinal na região dorsal de seu mesossoma, seguido de uma mancha triangular no prosoma (BRASIL, 2009).

Compreende-se por escorpionismo o quadro de envenenamento humano causado pela toxina escorpiônica, cuja gravidade e evolução variam amplamente desde casos leves até morte ou sequelas causadoras de incapacidade temporária para o trabalho e outras atividades habituais (BARBOSA, 2014). De acordo com a gravidade, os acidentes por escorpiões podem ser classificados em leves, moderados e graves (Quadro 1).

QUADRO 1 - Classificação quanto à gravidade dos acidentes escorpiônicos e tratamento.

\begin{tabular}{|c|c|c|c|}
\hline Classificação & Manifestações Clínicas & $\begin{array}{l}\text { Tratamento } \\
\text { Inespecífico }\end{array}$ & $\begin{array}{c}\text { Tratamento } \\
\text { Específico }\end{array}$ \\
\hline Leve & $\begin{array}{c}\text { Dor, edema, eritema, } \\
\text { sudorese }\end{array}$ & $\begin{array}{c}\text { Observação clínica, } \\
\text { anestésico local e/ou } \\
\text { analgésico }\end{array}$ & - \\
\hline Moderado & $\begin{array}{l}\text { Quadro local e uma ou } \\
\text { mais manifestações } \\
\text { como: náuseas, vômitos, } \\
\text { sudorese e sialorreia } \\
\text { discretas, agitação, } \\
\text { taquipnéia e taquicardia }\end{array}$ & $\begin{array}{c}\text { Internação hospitalar, } \\
\text { anestésico local e/ou } \\
\text { analgésico }\end{array}$ & $\begin{array}{c}3 \text { ampolas de } \\
\text { SAEEs }\end{array}$ \\
\hline
\end{tabular}




\begin{tabular}{|c|c|c|c|}
\hline Grave & $\begin{array}{c}\text { Além das manifestações } \\
\text { acima: vômitos profusos e } \\
\text { incoercíveis, sudorese e } \\
\text { sialorreia intensa, } \\
\text { prostração, convulsão, } \\
\text { coma, bradicardia, } \\
\text { insuficiência cardíaca, } \\
\text { edema agudo de pulmão, } \\
\text { choque. }\end{array}$ & $\begin{array}{c}\text { Internação em } \\
\text { Unidade de Terapia } \\
\text { Intensiva }\end{array}$ & $\begin{array}{c}6 \text { ampolas de } \\
\text { SAEEs }\end{array}$ \\
\hline
\end{tabular}

FONTE: Ministério da Saúde (Ofício Circular nº 04/ 2016 - CGDT/SVS, 2016.

Nos acidentes moderados e graves observa-se leucocitose com neutrofilia, hiperglicemia, hiperamilasemia, hipopotassemia e hiponatremia. Em casos graves a enzima creatinoquinase subunidades $M$ e $B(C K M B)$ e a troponina I (proteína altamente específica para o tecido miocárdico) podem estar aumentadas (PARDAL; GADELHA, 2010).

A toxina escorpiônica contém uma variedade de compostos, incluindo toxinas peptídicas mais ou menos potentes e seletivas que atuam sobre proteínas de canais iônicos que estão amplarmente distribuídas em muitos sistemas, tais como nervoso, esquelético e cardiovascular (ANDREOTTI; SABATIER, 2013).

Atua nas terminações nervosas desencadeando a liberação de mediadores químicos que são responsáveis por grande parte dos sintomas apresentados pelo paciente; age ainda estimulando a liberação de citocinas e prostaglandinas. As citocinas são liberadas por macrófagos em resposta inflamatória ao envenenamento (MACHADO, 2016).

Das 1.600 espécies conhecidas no mundo, apenas cerca de 25 são consideradas de interesse em saúde. No Brasil, onde existem cerca de 160 espécies de escorpiões, as responsáveis pelos acidentes graves pertencem ao gênero Tityus, que possui aproximadamente 170 espécies conhecidas (BORGES et al., 2010) que tem como característica, entre outras, a presença de um espinho sob o ferrão. As principais espécies capazes de causar acidentes graves são Tityus serrulatus, $T$. bahiensis, T. paraensis, e T. stigmurus, que corresponde ao principal agente causador de escorpionismo na região Nordeste do país (FREIRE et al., 2018). Também conhecido como escorpião amarelo, $T$. stigmurus pertence à família 
Buthidae, estando presente principalmente nos estados da Paraíba, Pernambuco, Alagoas, Sergipe e Rio Grande do Norte (ARAÚJO, 2016).

Relatou-se que o veneno produzido por $T$. stigmurus possui uma maior toxicidade em comparação com outros escorpiões do mesmo gênero, como $T$. serrulatus e T. bahiensis. O veneno produzido por estes escorpiões consiste em uma mistura complexa de compostos bioativos, que contém neurotoxinas e uma variedade de peptídeos de interesse farmacológico, incluindo hipotensinas e peptídeos antimicrobianos (FREIRE et al., 2018).

No Brasil, pode-se observar que as notificações envolvendo envenenamento por animais peçonhentos têm aumentado de forma extraordinária nos últimos anos. Segundo dados do SINAN, o número de ocorrências aumentou de 101.724 casos em 2007, para 221.004 em 2017, onde o Nordeste registrou aproximadamente 75.183 casos, entre estes, os acidentes com escorpião foram responsáveis por mais de 56 mil casos, seguido por ofidismo (acidente por envenenamento de serpente), com cerca de 7.247 (SINAN, 2019).

Diante do exposto, é importante ressaltar que, nesses acidentes, se faz necessária a procura imediata do serviço de saúde especializado para atenção às urgências clínicas, com o intuito de neutralizar rapidamente as toxinas inoculadas na vítima e implementar medidas de estabilização das condições vitais do acidentado (MESCHIAL et al., 2013). Além da assistência prestada, fica a cargo das unidades de saúde a notificação/comunicação da ocorrência à autoridade sanitária, pois mediante a avaliação destes dados são elaboradas as medidas de intervenção pertinentes (SANTANA; SUCHARA, 2015).

\section{RESULTADOS E DISCUSSÃO}

Entre janeiro e dezembro de 2017, notificou-se 56.100 casos de escorpionismo somente na Região Nordeste, seguido da Região Sudeste com 54.393 casos. As duas regiões concentraram $89,1 \%$ dos acidentes envolvendo 
escorpiões no país. Dos 56.100 casos, a maioria, 56,75\% ( $n=31.841)$ corresponde ao sexo feminino (Tabela 2 ).

O fato das mulheres corresponderem ao sexo com maior número de acidentes é justificado em razão de permanecerem maior tempo em suas residências, principalmente porque a maioria destas possuem ocupações como "aposentada", "do lar" e "doméstica", logo, estão mais relacionadas a atividades domésticas, que podem ser consideradas atividades de risco em relação a estes acidentes (MARQUES et al., 2015) principalmente a limpeza de locais propícios ao encontro de escorpiões, tornando-se mais vulneráveis.

Em relação à faixa etária, a faixa compreendida entre 20 a 39 anos (17.696; $31,5 \%)$ foi a mais afetada, o que difere com vários estudos que demonstram que crianças e idosos são os extremos etários mais afetados (FARIAS, 2014). Em relação à classificação do caso, a maioria foi identificada como "leve" ( $n=50.094$; $89,3 \%$ ), enquanto que os casos moderados ocorreram em 2.639 indivíduos (4,7\%), estes, por sua vez, necessitaram de tratamento específico com soroterapia antiescorpiônica (SAEEs), com uma média de 2 ampolas por paciente. O protocolo de tratamento soroterápico foi adotado conforme algoritmo de atendimento padronizado pelo Ministério da Saúde, que indica administração de soroterapia específica para quadros moderados e graves, onde há manifestações sistêmicas, sendo o número de doses adequado com a gravidade do caso (BRASIL, 2016).

Em relação ao número de casos, o Estado de Pernambuco foi o responsável pela maioria das notificações, ou seja, 14.668 casos registrados $(26,1 \%)$. O maior número de óbitos foi no estado da Bahia (16; 11,4\%); Alagoas apresentou a maior incidência, com cerca de 267 casos a cada 100.000 habitantes, e o Maranhão apresentou a maior taxa de Letalidade, com 36,1\%.

TABELA 2 - Perfil epidemiológico dos acidentes escorpiônicos, notificados pela Região Nordeste, entre janeiro e dezembro de 2017.

\begin{tabular}{lcc}
\hline Faixa Etária & $\mathbf{n}$ & $\mathbf{\%}$ \\
\hline 0 a 14 & 11.925 & $21,3 \%$ \\
15 a 19 & 4.517 & $8,1 \%$ \\
20 a 39 & 17.696 & $31,5 \%$ \\
40 a 59 & 14.224 & $25,4 \%$ \\
$>60$ & 7.727 & $13,8 \%$
\end{tabular}




\begin{tabular}{lcc} 
Ignorado & 11 & $0,0 \%$ \\
Subtotal & 56.100 & $100,0 \%$ \\
\hline Sexo & & \\
\hline Masculino & 24.252 & $43,2 \%$ \\
Feminino & 31.841 & $56,8 \%$ \\
Ignorado & 7 & $0,0 \%$ \\
Subtotal & 56.100 & $100,0 \%$ \\
\hline Classificação do caso & & \\
\hline Leve & 50.094 & $89,3 \%$ \\
Moderado & 2.639 & $4,7 \%$ \\
Grave & 252 & $0,4 \%$ \\
Ignorado & 3.115 & $5,6 \%$ \\
SubTotal & 56.100 & $100,0 \%$ \\
\hline Evolução do caso & & \\
\hline Cura & 51.123 & $91,1 \%$ \\
Perda de segmento/ignorado & 4.944 & $8,8 \%$ \\
Óbito & 33 & $0,1 \%$ \\
Total & 56.100 & $100,0 \%$ \\
\hline
\end{tabular}

Fonte: SINAN, 2020

A ocorrência destes acidentes na região urbana pode ser explicada pela alta densidade demográfica, crescimento desordenado e acúmulo de lixo favorecendo a proliferação de baratas, principal alimento do escorpião. A migração dos escorpiões de seu habitat natural para a zona urbana, ocorre devido à ocupação humana nas regiões originalmente habitadas por estes aracnídeos que, devido a seus hábitos criptozoicos, passaram a viver em amontoados de lixo e obras, com condições propícias de temperatura, umidade e disponibilidade de alimento para sobrevivência e reprodução durante todo o ano (BRASIL; BRITES-NETO, 2019; SANTANA; SUCHARA, 2015). 
GRÁFICO 1 - Número de casos e óbitos (\%) dos casos de escorpionismo, taxa de incidência (por 100 mil habitantes) e de letalidade (\%), de 2017, na região Nordeste.

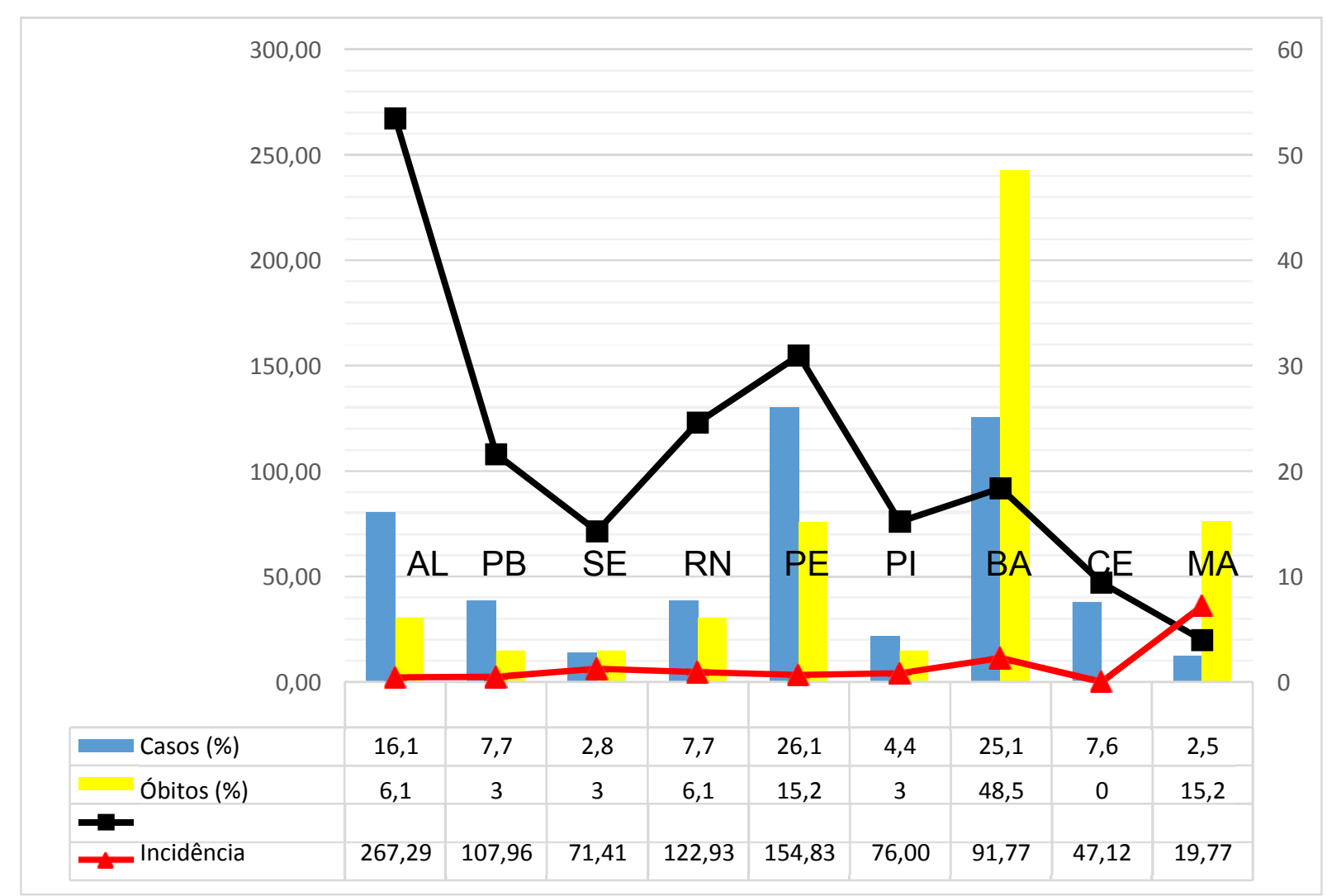

Fonte: SINAN, 2020.

A evolução para a cura foi constatada na maioria dos casos, o que corrobora com diversos estudos em outros estados brasileiros (ZANELLA, 2018; BARBOSA, 2016; FURTADO et al., 2016). A maioria dos casos tem evolução benigna, sendo as ocorrências graves e óbitos associados a crianças, idosos, portadores de doenças de base e à espécie $T$. serrulatus (ZANELLA, 2018).

\section{CONSIDERAÇÕES FINAIS}

O escorpionismo é um problema de saúde pública devido à elevada incidência em várias regiões do País, especialmente Nordeste e Sudeste, que 
juntos, correspondem a quase $90 \%$ dos casos notificados no Brasil. Verifica-se uma rápida progressão do número de casos nos últimos anos, ultrapassando o ofidismo em números absolutos; esse aumento reflete os altos índices de infestação por escorpiões na área urbana, onde estes animais têm encontrado condições favoráveis de sobrevivência.

Os acidentes por animais peçonhentos aumentam o número e o tempo de internação nos serviços de saúde, acarretando em alta ocupação de leitos, sobrecarga dos profissionais e resultando em maiores gastos para o Sistema Único de Saúde (SUS).

O Brasil avançou muito com relação ao controle dos acidentes por animais peçonhentos, instituindo o Programa Nacional de Controle de Acidentes por Animais Peçonhentos, cujos objetivos são: diminuir a letalidade dos acidentes ofídicos e escorpiônicos, através do uso adequado da soroterapia e a diminuição do número de casos através da educação em saúde.

\section{REFERÊNCIAS BIBLIOGRÁFICAS}

ALBUQUERQUE, P. C. C., GURGEL, I. G. D., GURGEL, A. M., AUGUSTO, L. G. S, SIQUEIRA, M. T., Sistemas de informação em saúde e as intoxicações por agrotóxicos em Pernambuco. Rev Bras Epidemiol, v. 18, n. 3, p. 666-678, 2015.

AMARAL, D. A.; HERNANDEZ, E. Ma M. In: OGA, SEIZE; CAMARGO, M. Ma DE A.; BATISTUZZO, J. A. DE O. Fundamentos de Toxicologia. 4. ed. São Paulo: Atheneu, 2014. $685 p$.

ANDREOTTI, N.; SABATIER, J.M. The Deciphered Genome of Mesobuthus martensii Uncovers the Resistance Mysteries of Scorpion to Its Own Venom and Toxins at the Ion Channel Level. Toxins. França, v.5, p. 2209-2211, 2013.

ARAÚJO, K. A. M. A., Estudo epidemiológico dos casos de acidentes por escorpião do Estado do Rio Grande do Norte (2007-2014). Dissertação de Mestrado. Programa de PósGraduação em Ciências Naturais e Biotecnologia). Universidade Federal de Campina Grande. Cuité, 2016.

BARBOSA, I. R., Aspectos do escorpionismo no estado do Rio Grande do Norte. Saúde.com, v. 10., n. 1, p. 43-53, 2014.

BARRETO, B. B., SANTOS. P. L., MARTINS, F. J. C., BARBOSA, N. R., RIBEIRO, L. C., LEITE, I. C. G. et al. Perfil epidemiológico dos acidentes ofídicos no município de Juiz de Fora MG no período de 2002-2007. Rev APS, v. 13, n. 2, p. 190-195, 2010.

BORGES, A., BERMINGHAM, E., HERRERA, N., ALFONZO, M. J., SANJUR, O. I., Molecular systematics of the neotropical scorpion genus Tityus (Buthidae): The historical biogeography 
and venom antigenic diversity of toxic Venezuelan species. Toxicon, v. 55 , n. 2, p. $436-454$, 2010.

BRASIL. Ministério da Saúde. Portaria $n^{\circ} 1.271$, de 6 de junho de 2014. Define a Lista Nacional de Notificação Compulsória de doenças, agravos e eventos de saúde pública nos serviços de saúde públicos e privados em todo o território nacional, nos termos do anexo, e dá outras providências. Diário Oficial da União 7 jun 2014.

BRASIL. Ministério da Saúde. Secretaria de Vigilância em Saúde. Nota Informativa $n^{\circ} 25$ de 2016. Informações da Coordenação-Geral de Doenças Transmissíveis (CGDT) referentes à nova abordagem ao tratamento em casos de acidentes por serpentes do grupo Bothrops ("jararacas") e por escorpiões, em situação de escassez de antivenenos. Disponível em:<www.dive.sc.gov.br/conteudos/agravos/publicacoes/TratamentoSerpentes.pdf>. Acesso em 22 Dezembro 2019.

BRASIL, J., BRITES-NETO, J., Avaliação da mobilidade de escorpiões Tityus serrulatus em área de infestação urbana de Americana, São Paulo, Brasil. J Health Biol Sci, v. 7, n. 1, p.2125, 2019.

BRASIL. Ministério da Saúde. Acidentes por animais peçonhentos. Disponível em:< http://portalms.saude.gov.br/saude-de-a-z/acidentes-por-animais-peconhentos>. Acesso em 21 Maio 2019.

CARMO, E. A., NERY, A. A., JESUS, C. S., CASOTTI, C. A., Internações hospitalares por causas externas envolvendo contato com animais em um hospital geral do interior da Bahia, 2009-2011. Epidemiol Serv Saude, v. 25, n. 1, p. 105-114, 2016.

FREIRE, M. C. L. C. et al., Molecular basis of Tityus stigmurus alpha toxin and potassium channel kV 1.2 interactions. J Mol Graph Model, v. 87, p.197-203, 2018.

IBGE. Instituto Brasileiro de Geografia e Estatística. Projeção da população do Brasil e das Unidades da Federação. Disponível em: https://www.ibge.gov.br/index.php. Acesso em 30 abr. 2019.

MACHADO, R. J. A., Caracterização Estrutural e avaliação da atividade biológica de uma nova hipotensina identificada no veneno do escorpião Tityus stigmurus. Tese de Doutorado. Departamento de Bioquímica. Universidade Federal do Rio Grande do Norte, Natal, 2016.

MARQUES, M. F. L., OLIVEIRA, I. C. C., ALBUQUERQUE, S. G. E. A., SILVA JUNIOR, S. V. S., OLIVEIRA, J. C. C., Perfil de idosos vítimas de acidente por escorpião. Anais CIEH, v. 2, n. $1,2015$.

MESCHIAL, W. C., MARTINS, B. F., REIS, L. M., BALLANI, T. S. L., BARBOZA, C. L., OLIVEIRA, M. L. F., Internações hospitalares de vítimas de acidentes por animais peçonhentos. Rev Rene, v. 14, n. 2, p. 311-319, 2013.

NAVES, M. M.; COSTA-CRUZ, J. M. High prevalence of Strongyloides stercoralis infection among the elderly in Brazil. Rev. Inst. Med. trop. S. Paulo, São Paulo, v. 55, n. 5, p. 309-313, 2013.

OLIVEIRA, H. F. A., COSTA, C. F., SASSI, R. Relatos de acidentes por animais peçonhentos e medicina popular em agricultores de Cuité, região do Curimataú, Paraíba, Brasil. Rev Bras Epidemiol, v. 16, n. 3, p. 633-643, 2013.

PARDAL, P. P. O., GADELHA, M. A. C., Acidentes com Animais Peçonhentos - Manual de Rotinas. $2^{\mathrm{a}}$ edição. Secretaria de Estado de Saúde Pública do Pará. Belém, 2010. 
PARISE, E. V., Vigilância e monitoramento dos acidentes por animais peçonhentos no município de Palmas, Tocantins, Brasil. Hygeia, v. 12, n. 22, p. 72-87, 2016.

SANTANA, V. T. P., SUCHARA, E. A., Epidemiologia dos acidentes com animais peçonhentos registrados em Nova Xavantina - MT. Rev Epidemiol Control Infect, v. 5, n. 3, p. 141-146, 2015.

SANTOS, P. L. C., MARTINS, F. J., VIEIRA, R. C. P. A, RIBEIRO, L. C., BARRETO, B. B., BARBOSA, N. R., Características dos acidentes escorpiônicos em Juiz de Fora - MG. Rev APS, v. 13, n. 2, p. 164-169, 2010.

SINAN. Sistema de Informação de Agravos de Notificação - Brasil. Acidentes por Animais Peçonhentos.
Disponível em: $<$ http://tabnet.datasus.gov.br/cgi/deftohtm.exe?sinannet/cnv/animaisbr.def>. Acesso em 20 Maio 2019.

ZANELLA, D. P., Características clínico-epidemiológicas do escorpionismo em Ipatinga, Minas Gerais, no período de 2010 a 2014. Dissertação de Mestrado. Instituto de Pesquisas Energéticas e Nucleares, Universidade de São Paulo. São Paulo, 2018. 